\title{
LOS ESTEROIDES ANABOLIZANTES ANDROGÉNICOS, RIESGOS Y CONSECUENCIAS
}

\section{ANABOLIC ANDROGENIC STEROIDS, RISKS AND CONSEQUENCES}

\author{
Rafael Ernesto Avella 1, Juan Pablo Medellín 2
}

\begin{abstract}
${ }^{1}$ Magister en Entrenamiento Deportivo, Instituto Manuel Fajardo; Especialista en Docencia Universitaria, Universidad Militar Nueva Granada, Docente Investigador de la Universidad de Ciencias Aplicadas y Ambientales U.D.C.A; Director del Grupo de Investigación en Actividad Física y Estilos de Vida Saludable; ravella@udca.edu.co 2 Estudiante Investigador de la Facultad de Ciencias del Deporte, Universidad de Ciencias Aplicadas y Ambientales U.D.C.A; Integrante del Grupo de Investigación en Actividad Física y Estilos de Vida Saludable; m_juanpablo9@hotmail.com
\end{abstract}

Rev. U.D.C.A Act. E Div. Cient. 15 (Supl. Olimpismo): 47 - 55, 2012

\section{RESUMEN}

El dopaje ha causado mucho daño en el mundo del deporte y de la actividad física, rechazado por deportistas, por entrenadores y por directivos, siendo perseguido, con el fin de ser erradicado de las justas deportivas, donde su empleo es causado por la presión de innumerables factores, que se presentan en el alto rendimiento; sin embargo, se desconoce el impacto y la popularidad que muchas de estas sustancias tienen en la gente del común; quienes buscan una perfección física, desconociendo las alteraciones funcionales, fisiológicas, bioquímicas y psicológicas que, en la mayoría de los casos, son irreversibles, con el único objetivo de encajar en los patrones de belleza, que impone la sociedad. Este trabajo recopila evidencia científica sobre los efectos de la ingesta de esteroides anabólicos androgénicos en el organismo, para generar conciencia en los potenciales usuarios y crear políticas de prevención, frente al consumo indiscriminado de dichas sustancias.

Palabras clave: Dopaje, deportes, alteraciones comportamiento, esteroides.

\section{SUMMARY}

Doping has caused much harm to the world of sport and physical activity, this has been rejected by athletes, coaches and managers, being chased to be eradicated in the just sports. However, it ignores the impact and popularity that many of these substances have in common people, who are seeking physical perfection, ignoring functional alterations, physiological, biochemical and psychological in most cases irreversible, with the sole purpose of fitting into the standards of beauty imposed by society. This paper collects evidence on the effects of the intake of anabolic androgenic steroids in the body, to generate awareness among potential users, and create policies for prevention against indiscriminate use of these substances.

Key words: Doping, sports, behavioral alterations, steroids.

\section{INTRODUCCIÓN}

El Dopaje, de acuerdo a la Agencia Mundial Antidopaje (WADA, 2009), está definido como "la administración o uso por parte de un atleta de cualquier sustancia ajena al organismo o cualquier sustancia fisiológica tomada en cantidad anormal, por una vía anormal con la sola intención de aumentar en un modo artificial y deshonesto su rendimiento en la competencia".

También se define, como "la violación de una o más de las reglas antidopaje descritas entre el artículo 2.1 al 2.8 del Código" (AMA, Código Mundial Antidopaje, 1984). Entre las violaciones de las reglas antidopaje, se encuentran: presencia en la muestra de una sustancia prohibida o sus metabolitos; uso o intento de uso de una sustancia prohibida; la no entrega de las muestras requeridas sin justificación alguna; la no disponibilidad del deportista en el período de test fuera de la competición; intento o manipulación del control antidopaje; posesión y/o tráfico de sustancias o métodos prohibidos y administrar o intentar administrar sustancias o métodos 
prohibidos a cualquier atleta (Gracia et al. 2009; Hildebrand, 2007). Para Colombia, la Ley 845 del 2003 dicta normas sobre prevención y lucha contra el dopaje (Constitución Política de Colombia, Diario Oficial 45.348).

Según Kayser \& Smith (2008), las políticas antidopaje se basan en cuatro razones, para luchar contra el dopaje:

- Igualar el nivel competitivo

- Proteger la salud de los deportistas

- Preservar la integridad del deporte

- Ser un "buen" ejemplo para la sociedad

En las definiciones anteriores, se tiene como requisito la competencia y el rendimiento deportivo; sin embargo, sustancias como los esteroides anabolizantes son utilizadas por un reducido grupo de deportistas y en cambio son de consumo habitual por un elevado número de usuarios, quienes participan en los gimnasios y no buscan la gloria deportiva sino mejorar su aspecto físico. Esto hace que el problema se salga de control, ya que las organizaciones nacionales e internacionales solo realizan controles y campañas de prevención en los deportistas de altos logros, que solo comprenden el $5 \%$ de la población que los utilizan, mientras el otro 95\%, lo constituyen las personas del común.

Frente a estas sustancias existen mitos y desinformación y lo único cierto es que son efectivas para elevar el rendimiento y mejorar la apariencia física, presentándose efectos secundarios que la mayoría de los usuarios desconoce y los cuales, tarde que temprano repercutirán sobre la salud y el bienestar.

Es necesario concientizar, informar, capacitar y prevenir el consumo de estas sustancias en la población, ya que en los últimos años el consumo se ha elevado en nuestro país.

\section{METODOLOGÍA PROPUESTA}

El ejercicio investigativo que se referencia a continuación corresponde a una revisión bibliográfica, que se basa en la metodología de tipo exploratoria, descrita, por Hernández et al. (2007), quien surge como resultado de búsqueda la temática del dopaje y, en especial, el problema de la utilización de los esteroides anabolizantes. El ejercicio responde a las características de diseño de investigación no experimental longitudinal evolutiva (Hernández et al. 2006).

Recopilación de la Información: Se realizó una búsqueda sistemática de la literatura especializada sobre el tema, de los últimos años, en revistas digitales e impresas, en libros técnicos y consultas con expertos.

Para la revisión sistemática, se organizó una búsqueda de material teniendo en cuenta su accesibilidad en las siguien- tes fuentes: 1) Determinar las palabras claves para la búsqueda en base de datos (Fuentes de información, fundamental Medline, Cochrane Library, Scielo, capítulos de libros o revistas indexadas; bases de datos disponibles en internet: Medline - PubMed (National Library of Medicine), Cochrane data base of Systematic Reviews, Doyma, Scielo, Biblioteca Virtual de Salud, ProQuest. 2) Buscadores: Mozilla Firefox. 3) Páginas web de los organismos nacionales e internacionales, relacionados con el control del dopaje.

\section{RESULTADOS Y DISCUSIÓN}

"El hombre siempre ha querido mejorar sus capacidades y su figura, es así como, a través de los tiempos siempre ha buscado la fórmula mágica que preserve la juventud, la vitalidad, con un mínimo de esfuerzo" (Rodríguez, 1992).

Actualmente, la sociedad exige cada vez más rendimiento a nivel intelectual, físico y laboral; creando nuevos modelos y súper estrellas e ídolos deportivos, quienes ganan millones por avisos y por reportajes publicitarios.

Los deportistas aficionados, se encuentran envueltos en este comercio que promociona sustancias y productos para alcanzar el éxito y la fama. En los gimnasios, se opta por seguir los consejos de los instructores y los entrenadores, quienes son poseedores de un gran físico que, en teoría, respaldan su conocimiento, pero en ocasiones no es cierto y se termina recibiendo orientaciones sin fundamento científico, consumiendo sustancias para acortar el camino en la consecución de los objetivos, sucumbiendo a tentaciones que, a largo plazo, solo causarán cambios y efectos a veces irreversibles, que perjudican al usuario y a sus familias.

Por presión social, se crea la necesidad de obtener el cuerpo perfecto, lo cual, genera desórdenes frente a la percepción de la corporeidad, es así, que trastornos como la bulimia y la anorexia se hacen presentes, en las mujeres y la vigorexia (anorexia inversa) o dismorfia muscular, en los hombres, términos considerados como lo contrario de la anorexia nerviosa, que provoca alteraciones alimenticias y crisis sicológicas, frente a la realidad. La vigorexia o dismorfia muscular es una forma de trastorno dismórfico corporal (Pope et al. 1997), que se basa en una preocupación patológica con la relación musculatura/delgadez, donde los individuos se comprometen en la búsqueda patológica de un hiper-mesomórfico físico (musculatura extrema), obligándose obsesiva y compulsivamente a participar en actividades para alcanzar tan altos niveles (Dawes \& Mankin, 2004; Pope et al. 2000).

Una de las actividades más populares practicada por deportistas de rendimiento y aficionados es el entrenamiento en los gimnasios, donde existe la cultura que promueve el 
desarrollo de la fuerza y la consecución de grandes músculos; esto conlleva, a querer obtener resultados a corto plazo y con mínimo esfuerzo, apareciendo la utilización de esteroides anabólicos androgénicos.

Los esteroides anabólicos androgénicos son derivados sintéticos de la testosterona (Haupt \& Rovere, 1984; Kicman \& Gower, 2003; García, 2002), que estimulan la producción celular de proteínas, provocando un aumento en el tamaño muscular (Kuhn et al. 2003; Bhasin et al. 1996), generado por un aumento de la sección transversal de la fibra (Sheffield-Moore et al. 1999; Bhasin et al. 1996) y un desarrollo de las capacidades físicas condicionales (fuerza, velocidad, resistencia, flexibilidad) (González Aramendi, 2008).

El cuerpo humano es capaz de producir 600 tipos diferentes de esteroides anabólicos androgénicos (Yesalis \& Cowart, 1999), generando dos efectos en el organismo: el anabólico, con el cual se logra la construcción o aumento de los tejidos (Sturmi \& Diorio, 1998), que se encuentra mediado por los receptores de andrógenos en el musculo (Sheffield-Moore et al. 1999) y el efecto androgénico, que origina efectos "masculinizantes" (Stedman's Electronic Medical Dictionary, 1998), mucho más evidentes en las mujeres.

Los esteroides anabólicos androgénicos, se pueden clasificar teniendo en cuenta diferentes características: la vía de administración, la duración del efecto y el objetivo a conseguir. Según la vía de administración, se catalogan en: a) Orales, que se caracterizan por soportar los ácidos estomacales y las enzimas hepáticas; su absorción se da a través del tracto gastrointestinal; su duración en el organismo es corta, por lo que se hace necesario tomar varias veces al día; se utilizan con el objetivo de desarrollar la fuerza máxima y se considera que tienen una alta toxicidad y efectos bastantes negativos, a mediano plazo (Emblenton \& Thorne, 2004). b) Inyectables, que se aplican intramuscularmente y se dividen en los que tienen base de agua, los cuales, tiene una vida media de 1 a 2 semanas y permiten combinaciones con otros esteroides; su nivel de toxicidad es bajo; se usan en la fase de definición muscular y sus efectos negativos se presentan a largo plazo y bajo la utilización de grandes cantidades. Los inyectables a base de aceite están preparados con una base de aceite de sésamo y alcohol, que soporta la concentración de esteres, que van de 25 a $250 \mathrm{mg} / \mathrm{mL}$, su duración es larga, de 2 a 4 semanas, se absorben lentamente a través del torrente sanguíneo y son utilizados para el incremento de la masa muscular. c) Los tópicos, se encuentran en parches, ungüentos, lociones, geles y cremas o pomadas; su periodo de vida es corto y se ubican en el mercado con diferentes concentraciones; se absorben por la dermis, permite la utilización en zonas específicas, de acuerdo a los objetivos que requiera; tienen efectos negativos a largo plazo, con riesgo de producir alteraciones cutáneas; otro inconveniente que presentan es la trasmisión por contacto; los factores que determinan su absorción y su eficacia son: la cantidad de sustancia que se absorbe en forma percutánea (Sáez, 1997) (dependiente del esteroide y sus propiedades fisicoquímicas, de la solubilidad del mismo y su concentración en el vehículo, el tipo de ingredientes del vehículo y, finalmente, del sitio y el estado de la piel en la que se aplique (Topert, 1988; Tauber, 1994; Dupuis et al. 1984; Krochmal et al. 1989) y la tasa de absorción hacia la circulación.

Otra clasificación se puede dar por el tiempo de acción en el organismo, donde se encuentran las sustancias de acción rápida, generalmente, utilizadas antes de la competencias y que tienen un efecto de minutos u horas, teniendo como objetivo enmascarar otros esteroides o poner a punto al deportista para su evento y los de acción a largo plazo, que se usan en períodos alejados de la competencia y tienen como objetivo la regeneración celular y el aumento de la masa muscular, que dependerá de la dieta y el entrenamiento; con éstos, se aumenta la fuerza, pero no la capacidad aeróbica de trabajo (Catlin et al. 1987; Hallagan et al. 1989; Haupt \& Rovere, 1984; Olsson, 1974).

El objetivo de los esteroides anabolizantes androgénicos no solamente está orientado a elevar el rendimiento deportivo o mejorar la apariencia física, ya que su uso en la medicina incluye el tratamiento de diversas enfermedades, como: osteoporosis, anemia aplástica, carcinoma de mama, quemaduras (Demling, 2005), endometriosis, daño en el miocardio, hipogonadismo, sarcopenia (Johansen et al. 1999; 2006), VIH (Hengge et al. 1996; Gold et al. 2006), EPOC (Ferreira et al. 1998), Isquemia Cardiaca (Liu et al. 2003), mielofibrosis, púrpura trombocitopenica (García, 2002), profilaxis a largo plazo del angioedema, criofibrinogenemia (Melnik et al. 2007) entre otras. La prescripción y la utilización de estas sustancias está científicamente programada por médicos especialistas, sin embargo, muchos deportistas y practicantes del culturismo los usan indiscriminadamente, desconociendo los efectos adversos que tienen en el organismo, a corto, mediano o largo plazo. Los efectos más comunes, se resumen en la tabla 1 y 2.

Los esteroides anabolizantes lesionan de diversas maneras a los individuos que los utilizan. Dependiendo del órgano que se afecte, se dividen en: a) Hepáticos: la hepatotoxicidad se refleja en la elevación de la función hepática (Haupt \& Rovere, 1984; Freed et al. 1975; Sader et al. 2001) y en el desarrollo de patologías, como el carcinoma y la hepatitis coléstatica. b) Cardiovasculares: el efecto provocado en el perfil lipídico involucra la elevación de la lipoproteína de baja densidad (LDL) y un descenso de la concentración de la lipoproteína de alta densidad (HDL) (Hickson et al. 1989; Sader 
Tabla 1. Efectos secundarios de los EAA sobre el organismo.

\begin{tabular}{|c|c|}
\hline Hepáticos & $\begin{array}{l}\text { - Elevaciones de función hepática (hepatotoxicidad) } \\
\text { - } \quad \text { El cáncer de hígado }\end{array}$ \\
\hline Cardiovasculares & $\begin{array}{ll}\text { - } & \text { Disminución del colesterol HDL } \\
\text { - } & \text { El aumento de LDL } \\
\text { - } & \text { Aumento del colesterol total de } \\
\text { - } & \text { Disminución de los triglicéridos } \\
\text { - } & \text { Retención de líquidos (presión arterial elevada) } \\
\text { - } & \text { La hipertrofia cardiaca }\end{array}$ \\
\hline $\begin{array}{l}\text { Reproductivos y } \\
\text { Endocrinos }\end{array}$ & $\begin{array}{ll}\text { - } & \text { Disminución de la LH } \\
\text { - } & \text { Disminución de la FSH } \\
\text { - } & \text { Disminución del funcionamiento de tiroides }\end{array}$ \\
\hline Dermatológicos & $\begin{array}{ll}\text { - } & \text { Cabello graso } \\
\text { - } & \text { Piel grasa } \\
\text { - } & \text { Alopecia } \\
\text { - } & \text { Quistes sebáceos } \\
\text { - } & \text { Aumento de la incidencia del acné }\end{array}$ \\
\hline Psiquiátricos & $\begin{array}{ll}\text { - } & \text { Cambios de humor } \\
\text { - } & \text { Posible agresión } \\
\text { - } & \text { Posible hostilidad } \\
\text { - } & \text { Dependencia y / o adicción }\end{array}$ \\
\hline
\end{tabular}

FSH = hormona estimulante del folículo; HDL = lipoproteína de alta densidad;

LDL = lipoproteína de baja densidad; LFT = pruebas de función hepática, la LH = la hormona luteinizante.

Fuente: Falduto, 1989; Kutscher et al. 2002.

Tabla 2: Efectos secundarios de los EAA por género.

\begin{tabular}{|l|ll|}
\hline \multirow{4}{*}{ En los hombres } & - & Disminución de la espermatogénesis \\
& - & Morfología de los espermatozoides anormales \\
& - & Feminización de los machos \\
& - & Disminución del tamaño de los testículos \\
\hline & - & Hirsutismo \\
En las mujeres & - & Engrosamiento de la voz \\
& - & Hipertrofia del clítoris \\
& - & Disminución de la masa de mama \\
& - & Camenorrea \\
\hline
\end{tabular}

Fuente: Perry et al. 1999; Kutscher et al. 2002.

et al. 2001; Glazer, 1991), lo que podría desarrollar arteriosclerosis; el incremento de la concentración de colesterol sérico es un factor de riesgo de la aparición de trombosis, así mismo, la estimulación de la agregación plaquetaria y, por ende, mayor coagulación enzimática puede causar vasoespasmo coronario; el aumento de la presión arterial (Freed 
et al. 1975; Riebe et al. 1992) viene como resultado del incremento de la volemia y la retención de líquidos; el uso de esteroides anabólicos androgénicos incrementa el ritmo cardiaco, lo que llevará a una hipertrofia del ventrículo izquierdo y así provocar una disminución máxima del consumo de oxígeno, la remodelación del corazón, lo que aumentará el riesgo de sufrir una miocardiopatía, con posibilidad de padecer arritmias (Hall et al. 2005), un paro cardiaco (Sullivan et al. 1998; Hickson et al. 1989; Koenig et al. 1982) o un accidente cerebrovascular (Dotson \& Brown, 2007); también se presenta una disminución de los triglicéridos (Blue \& Lombardo, 1999; Pardrige, 1981), acompañada de eritrocitosis (García, 2002). c) Reproductivos y endocrinos: el exceso en el consumo de esteroides anabólicos androgénicos tendrá como resultado una disminución de la segregación de las hormonas luteinizante (LH) y foliculoestimulante (FSH), necesarias para la espermatogénesis, lo que genera hipogonadismo hipogonadotrifico (atrofia testicular) (Gill, 1998; Jarow \& Lipshultz, 1990; MacIndoe et al. 1997; Alen et al. 1985a y b; Fujioka et al. 1987; Mathur, 1981; Ruedi, 1983; Zitzmann et al. 2002; Sader et al. 2003); los espermatozoides sufren una disminución en la densidad, en el número y la motilidad, presentando una morfología anormal (Yesalis \& Bahrke, 1995; Hickson et al. 1989; Holma, 1977); además, se puede producir una oligospermia (bajo volumen de semen), produciendo infertilidad (Turek et al. 1995). Otro factor asociado es la denominada aromatización de los esteroides, donde el exceso de testosterona es convertida en estrógenos, que genera una feminización en el organismo (Hickson et al. 1989), que se manifestará en la ginecomastia y cambio en el tono de la voz, (Kutscher et al. 2002; Phillips, 1990). d) Psicológicos: dentro de los efectos más descritos en la literatura especializada, se registra el incremento de la agresividad, la irritabilidad, la ansiedad, la euforia y la depresión (Parroit et al. 1994; Kouri et al. 1995; Tricker et al. 1996; Pope et al. 2000; Matsumoto, 1990; González Aramendi, 2008; Choi et al. 1990; Moss et al. 1992); en algunos casos, se presenta la aparición de síndromes maniacos e hipomaniacos, reflejándose episodios paranoides y de alucinaciones, ocasionando suicidios, aberraciones sexuales, entre otros trastornos, siendo adictivos y generando dependencia (Sader et al. 2001; Clark et al. 2003).

Los efectos en las mujeres son más complicados y, en la mayoría de los casos, irreversibles; dentro de éstos, se manifiestan: hirsutismo (crecimiento excesivo del vello), acné, engrosamiento de la voz, hipertrofia del clítoris, disminución de la masa de la mama, disminución de la menstruación o amenorrea, aumento del apetito y calvicie de patrón masculino (Straus et al. 1985). Los efectos endocrinos, se revelan en la disminución de la función de la hormona tiroides (Alen et al. 1987; Shimizu, 1988) y, por ende, una baja producción de la tiroxina (Blue \& Lombardo, 1999), hiperinsulinemia y alteración de la tolerancia de la glucosa (Lasarre et al. 1974). También están los efectos dermatológicos: se elevan los niveles de propionibacterium: acnés, ácidos grasos libres y colesterol en la dermis, originando cabello y piel grasos, alopecia, quistes sebáceos e hipertrofia de las glándulas sebáceas (Blue \& Lombardo, 1999). A nivel de músculo-esquelético: la utilización de estas sustancias en adolescentes genera una osificación temprana (cierre prematuro de los centros de crecimiento de los huesos largos), con detención del crecimiento, además de incrementarse las lesiones articulares y tendinosas (González Aramendi, 2008).

Los efectos positivos y negativos dependerán del tipo y especificidad de la droga, la dosis, la vía, la frecuencia, la forma y el tiempo total de la administración, la edad, el género, la dieta, el entrenamiento, la herencia, la experiencia en la actividad y la situación físico-psíquica del atleta, siendo su acción diferente en cada individuo (Wilson, 1988).

Para el consumo y la utilización de los esteroides anabólicos androgénicos, se utilizan los denominados ciclos, que son periodos de tiempo, en los cuales, se programa el manejo de diferentes combinaciones de esteroides anabolizantes androgénicos, que responden a una organización de tiempo de 8 hasta 20 semanas.

Dentro de los ciclos más conocidos y aplicados, se encuentran (Phillips, 1990; Emblenton \& Thorne, 2004):

- Diamante: Se combinan esteroides en tabletas con ampolletas, se hace un aumento progresivo en la ingesta de los orales y el objetivo primordial es el incremento de la masa muscular y la fuerza máxima.

- Descendente: Se utilizan dos inyectables, con tabletas, iniciando con altas cantidades que van disminuyéndose progresivamente hasta completar el periodo; su objetivo es el incremento de la masa muscular.

- Repetido Alterno: Se combinan toma oral, inyectable y hormona de crecimiento, realizando alternadamente estas ingestas durante un periodo; su objetivo se basa en el incremento de la masa muscular y la fuerza máxima.

- Mini ciclo: El primer periodo se combinan toma oral e inyectable, aumentado progresivamente y luego disminuyéndola; seguidamente, se utiliza hormona de crecimiento para después repetir el primer periodo; finalmente, se aplicará, de nuevo, la hormona de crecimiento; se ejecutará para obtener definición muscular.

- Ataque: Se inicia con un periodo de toma oral, en seguida, se usa toma inyectable y, luego, toma oral, siendo un periodo largo y, al final, se administra hormona de crecimiento; su práctica será conducido a la obtención de masa muscular. 
- Pre competitivo: Se utilizan quemadores de grasa, junto con pequeña toma inyectable y hormona de crecimiento, usándose para la definición muscular.

- Femenino: Se usa baja concentración de toma inyectable, siendo la menos perjudicial para la salud, obteniéndose disminución en el porcentaje de grasa y aumento de la masa muscular.

\section{CONCLUSIÓN}

Queda de manifiesto que los esteroides anabolizantes androgénicos son hormonas muy efectivas para el desarrollo de las capacidades físicas y la hipertrofia muscular, con éstas, se consiguen resultados a muy corto tiempo; sin embargo, los prejuicios que se sufren a corto, mediano y largo lapso afectan, de manera perjudicial, el buen funcionamiento del organismo y es donde surge el interrogante si se justifica la utilización de dichas sustancias para la obtención de "Super resultados", sin el esfuerzo meritorio, poniendo en riesgo la salud.

Conflicto de Intereses: El manuscrito fue preparado y revisado con la participación de todos los autores, quienes declaramos que no existe ningún conflicto de intereses que ponga en riesgo la validez de los resultados.

\section{BIBLIOGRAFÍA}

1. ALEN, M.; RAKKILA, P.; REINILA, M.; VIHKO, R. 1987. Androgenic-anabolic steroid effects on serum thyroid, pituitary and steroid hormones in athletes. Am. J. Sport Med. 15:357-361.

2. ALEN, M.; RAKKILA, P.; MARNLEML, J. 1985a. Serum lipide in power athletes self-administering testosterone and anabolic steroids. Int. J. Sport Med. 6(1):139-144.

3. ALEN, M.; REINILA, M.; VIHKO, R. 1985b. Response of serum hormones to androgen administration in power athletes. Med. Sci. Sports Exerc. 17(3):354359.

4. AMERICAN COLLEGE OF SPORT MEDICINE AMA. 1984. Position stand on the use of Anabolic Androgenic Steroids in Sport. Sport Medicine Bull. 19:13-18.

5. BHASIN, S.; STORER, T.W.; BERMAN, N.; CALLEGARI, C.; CLEVENGER, B.; PHILLIPS, J.; BUNNELL, T.J.; TRICKER, R.; SHIRAZI, A.; CASABURI, R. 1996. The effects of supraphysiologic doses of testosterone on muscle size and strength in normal men. N. Engl. J. Med. 335(1):1-7.

6. BLUE, J.G.; LOMBARDO, J.A. 1999. Steroids and steroid-like compounds. Clin. Sports Med. 18:667689.

7. CATLIN, D.H.; KAMMERER, R.C.; HATTON, C.K.; SEKERA, M.H.; MERDINK, J.M. 1987. Analytical chemistry at the games of the XXIII rd Olympiade in Los Angeles. Clin. Chem. 33:319-327.

8. CHOI, P.Y.; PARROTT, A.C.; COWAN, D. 1990. High-dose anabolic steroids in strength athletes: effects upon hostility and aggression. Hum Psychopharmacol. 5(4):349-356.

9. CLARK, A.S.; HENDERSON, L.P. 2003. Behavioral and physiological responses to anabolic-androgenic steroids. Neurosci. Biobehav. Review. 27(5):413-436.

10. CONSTITUCIÓN POLÍTICA DE COLOMBIA, Ley 845 del 2003, Diario Oficial 45.348, 21 de octubre del 2003.

11. DAWES, J.; MANKIN, T. 2004. Muscle dysmorphia. J. Strength Condit. Res. 26:24-25.

12. DEMLING, R.H. 2005. The role of anabolic hormones for wound healing in catabolic states. J. Burn Wounds. 4:e2.

13. DOTSON, J.L.; BROWN, R.T. 2007. The history of the development of anabolic-androgenic steroids. Pediatr. Clin. N. Am. 54(4): 761-769.

14. DUPUIS, D.; ROUGIER, A.; ROGUET, R.; LOTTE, C.; KALOPISSIS, G. 1984. In vivo relationship between horny layer reservoir effect and percutaneous absorption in human and rat. J. Invest. Dermatol. 82:353-356.

15. EMBLENTON, P.H.; THORNE, G. 2004. Anabolic Primer. Ed. Olympus Sport Nutricion. Barcelona. 45p.

16. FALDUTO, M.T. 1989. Adverse effects of anabolic steroids. Med. Toxicol. Adverse Drug Exp. 4:254271.

17. FERREIRA, I.M.; VERRESCHI, I,T.; NERY, L.E.; GOLDSTEIN, R.S.; NOE ZAMEL, N.; DINA BROOKS, D.; JARDIM, J.R. 1998. The influence of 6 months of 
oral AAS on body mass and respiratory muscles in undernourished COPD patients. Chest. 114:19-28.

18. FREED, D.L.; BANKS, A.J.; LONGSON, D.; BURLEY, D.M. 1975. Anabolic steroids in athletics: crossover double-blind trial on weightlifters. BMJ. 2:471-473.

19. FUJIOKA, M.; SHINOHARA, Y.; BABA, S.; IRIE, M.; INONUE, K. 1987. Acute suppression of endogenous testosterone levels by exogenous testosterone in normal men. Life Sci. 41:945-949.

20. GARCÍA MALDONADO, G. 2002. Complicación psiquiátricas por el uso de esteroides anabólicos. Rev. Med. IMSS. 40(2):103-112.

21. GILL, G.V. 1998. Anabolic steroid induced hypogonadism treated with human chorionic gonadotropin. Postgrad. Med. J. 74:45-46.

22. GLAZER, G. 1991. Arthrogenic effects of anabolic steroids on serum lipid levels. Arch. Intern. Med. 151:1925-1933.

23. GOLD, J.; BATTERHAM, M.J.; REKERS, H.; HARMS, M.K.; GEURTS, T.B.; HELMYR, P.M.; SILVA DE MENDONÇA, J.; FALLEIROS CARVALHO, L.H.; PANOS, G.; PINCHERA, A.; AIUTI, F.; LEE, C.; HORBAN, A.; GATELL, J.; PHANUPHAK, P.; PRASITHSIRIKUL, W.; GAZZARD, B.; BLOCH, M.; DANNER, S.A. 2006. Effects of nandrolone deeanoate compared with placebo or testosterone on HIV-associated wasting. HIV Med. 7(3):146-155.

24. GONZÁLEZ ARAMENDI, J.M. 2008. Uso y abuso de esteroides anabolizantes. BIBLID. 26:185-197.

25. GRACIA-MARCO, L.; REY LÓPEZ, J.P.; CASAJÚS MALLÉN, J.A. 2009. El dopaje en los Juegos Olímpicos de verano (1968-2008). Apuntes Med. Sports. 44:66-73.

26. HALL, R.C.; CHAPMAN, M.G. 2005. Psychiatric complications of anabolic steroid abuse. Psychosomatics. 46(4):285-290.

27. HALL, R.C.W.; HALL, R.C.W. 2005. Abuse of Supraphysiologic Doses of Anabolic Steroids. Southern Med. J. 98(5):550-555.

28. HALLAGAN, J.B.; HALLAGAN, L.F.; SUYAER, M.B. 1989. Anabolic-androgenic steroid use by athletes». N. Eng. J. Med. 321(15):1042-1045.
29. HAUPT, H.A.; ROVERE, G.D. 1984. Anabolic steroids: a review of the literature. Am. J. Sports Med. 12:469484.

30. HENGGE, U.R.; BAUMANN, M.; MALEBA, R.; BROCKMEYER, N.H.; GOOS, M. 1996. Oxymetholone promotes weight gain in patients with advanced human inmunodeficiency virus (HIV-1) infection. Br. J. Nutr. 75:129-138.

31. HERNÁNDEZ, R.; FERNÁNDEZ, C.; BAPTISTA, P. 2006. Metodología de la investigación. Ed. Mc. Graw Hill. México. 216p.

32. HERNÁNDEZ, R.; FERNÁNDEZ, C.; BAPTISTA, P. 2007. Fundamentos de metodología de la investigación. Ed. Mc. Graw Hill. México. 59p.

33. HICKSON, R.C.; BALL, K.L.; FALDUTO, M.T. 1989. Adverse effects of anabolic steroids. Med. Toxicol. Adverse Drug Exp. 4:254-271.

34. HILDEBRAND, R.L. 2007. El programa mundial antidopaje y el médico de atención primaria. Pediatr. Clin. N. Am. 54:701-711.

35. HOLMA, P.K. 1977. Effects of an anabolic steroid (metandienone) on spermatogenesis. Contraception. 15:151-162.

36. JAROW, J.P.; LIPSHULTZ, L.I. 1990. Anabolic steroidinduced hypogonadotropic hypogonadism. Am. J. Sports Med. 18:429-431.

37. JOHANSEN, K.L.; MULLIGAN, K.; SCHAMBELAN, M. 1999. Anabolic effects of nandrolone decanoate in patients receiving dialysis: a randomized controlled trial. JAMA. 281:1275-1281.

38. JOHANSEN, K.L.; PAINTER, P.L.; SAKKAS, G.K.; GORDON, P.; DOYLE, J.; SHUBERT, T. 2006. Effects of resistance exercise training and nandrolone decanoate on body composition and muscle function among patients who receive hemodialysis: a randomized, controlled trial. J. Am. Soc. Nephrol. 17:2307-2314.

39. KAYSER, B.; SMITH, A. 2008. Globalisation of antidoping: the reverse side of the medal. BMJ.337:a584.

40. KICMAN, A.T.; GOWER, D.B. 2003. Anabolic steroids in sport: biochemical, clinical and analytical perspectives. Ann. Clin. Biochem. 40:321-356. 
41. KOENIG, H.; GOLDSTONE, A.; LU, C.Y. 1982. Testosterone-mediated sexual dimorphism of the rodent heart: ventricular lysosomes, mitochondria, and cell growth are modulated by androgens. Circulation Res. 50:782-787.

42. KOURI, E.M.; LUKAS, S.E.; POPE, H.G. Jr.; OLIVA, P.S. 1995. Increased aggressive responding in male volunteers following the administration of gradually increasing doses of testosterone cypionate. Drug Alcohol Depend. 40(1):73-79.

43. KROCHMAL, L.; WANG, J.C.T.; PATEL, B.; RODGERS, J. 1989. Topical corticosteroid compounding. Effects on physicochemical stability and skin penetration rate. J. Am. Acad. Dermatol. 21:979-984.

44. KUHN, C.; SWARTZWELDER, S.; WIÑSON, W. 2003. Anabolizantes, estimulantes y calmantes en la práctica deportiva, Barcelona, Ed. Paidotribo. 77p.

45. KUTSCHER, E.; LUND, B.; PERRY, P. 2002. Anabolic steroids a review for the clinician. Sports Med. 32(5):285-296.

46. LASARRE, C.; GIRARD, F.; DURN, J.; RAYNAUD, J. 1974. Kinetics of human growth hormone during submaximal exercise. J. Appl. Physiol. 37(6): 826830.

47. LIU, P.Y.; DEATH, A.K.; HANDELSMAN, D.J. 2003. Androgens and cardiovascular disease. Endocr. Rev. 24:313-340.

48. MacINDOE, J.H.; PERRY, P.J.; YATES, W.R.; HOLMAN, T.L.; ELLINGROD, V.L.; SCOTT, S.D. 1997. Testosterone suppression of the HPT axis. J. Invest. Med. 45: 441-447.

49. MATHUR, R.S. 1981. Plasma androgens and sex hormone binding globulin (SHBG) in the evaluation of hirsute females. Fert. Ster. 35:29-35.

50. MATSUMOTO, A.M. 1990. Effects of chronic testosterone administration in normal men: safety and efficacy of high dosage testosterone and parallel dosedependent suppression of luteinizing hormone, follicle stimulating hormone, and sperm production. J. Clin. Endocr. Metab. 70:282-287.

51. MELNIK, B.; JANSEN, T.; GRABBE S. 2007. Abuse of anabolic-androgenic steroids and bodybuilding acne: an underestimated health problem. J. Dtsch Dermatol. Ges. 5(2):110-117.

52. MOSS, H.B.; PANZAK, G.L.; TARTER, R.E. 1992. Personality, mood and psychiatric symptoms among anabolic steroid users. Am. J. Addict. 1(4):315-324.

53. OLSSON, A. 1974. Effects of oxandrolone on plasma lipoproteins and the intraveous fat tolerance in man. Atheroscler. 19: 337-346.

54. PARDRIGE, W.H. 1981. Transport of protein bound hormones into tissues in vivo. Endrocr. Rev. 2:103 123.

55. PARROIT, A.C.; CHOI, P.Y.; DAVIES, M. 1994. Anabolic .steroid use by amateur athletes: effects upon psychological mood states. J. Sports Med. Phys. Fitness. 34:292-298.

56. PERRY, P.J.; MacINDOE, J.; HOLMAN, T.; ELLINGROD, V. 1999. Psychosexual effects of three doses of testosterone cycling in normal men. Biol. Psychiatry. 45:254-260.

57. PHILLIPS, N. 1990. Guía de referencia anabólica. Ed. Mile High Publ., California. p.90-97.

58. POPE, H.G. Jr.; KOURI, E.M.; HUDSON, J.I. 2000. Effects of supraphysiologic doses of testosterone on mood and aggression in normalmen: a randomized controlled trial. Arch. Gen. Psychiatry. 57:133-140.

59. POPE, H.G. Jr.; GRUBER, A.J.; CHOI, P.; OLIVARDIA, R.; PHILLIPS, K.A. 1997. Muscle dysmorphia, an unrecognised form of body dysmorphic disorder. Psychosomatics. 38:548-557.

60. RIEBE, D.; FERNHALL, B.; THOMPSON, P.D. 1992. The blood pressure response to exercise in anabolic steroid users. Med. Sci. Sports Exerc. 24:633-637.

61. RODRÍGUEZ BUENO, C. 1992. Dopaje. Ed. McGraw Hill, Madrid. p.3-17.

62. RUEDI, B. 1983. Diagnostic strategy in hyperandrogenic syndrome. Horm. Res. 18:117-124.

63. SADER, M.A.; GRIFFITHS, K.A.; McCREDIE, R.J.; HANDELSMAN, D.J.; CELERMAJER, D.S. 2001. Androgenic anabolic steroids and arterial structure and function in male bodybuilders. J. Am. Coll. Cardiol. 37:224-230. 
64. SADER, M.A.; GRIFFITHS, K.A.; SKILTON, M.R.; WISHART, S.M.; HANDELSMAN, D.J.; CELEMAJER, D.S. 2003. Physiological testosterone replacement and arterial endothelial function in men. Clin. Endocrin. 59:62-67.

65. SÁEZ DE OCARIZ, M.M. 1997. Esteroides tópicos. Dermatol. Rev. Mex. 41:184-189.

66. SHEFFIELD-MOORE, M.; URBAN, R.J.; WOLF, S.E.; JIANG, J.; CATLIN, D.H.; HERNDON, D.N.; WOLFE, R.R. 1999. Short-term oxandrolone administration stimulates net muscle protein synthesis in young men. J. Clin. Endocrin. Metab.; 84(8):2705-2711.

67. SHIMIZU, K. 1988. Synthesis of deuterium-labeled 17hydroxi-progesterone suitable as an internal standard for isotope dilution mass spectrometry. Steroids. 51:283-297.

68. STRAUS, R.H.; LIGGETT, M.; LANESE, R.R. 1985. Anabolic steroid use and perceived effects in ten weight-trained women athletes. JAMA. 253.:28712873.

69. STURMI, J.E.; DIORIO, D.J. 1998. Anabolic agents. Clin Sports Med. 17:261-282.

70. SULLIVAN, M.L.; MARTÍNEZ, C.M.; GENNIS, P.; GALLAGHER, E.J. 1998. The cardiac toxicity of anabolic steroids. Prog Cardiovasc. Dis. 41:1-15.

71. TAUBER, U. 1994. Pharmacokinetics and "bioactivation" of MPA. J. Eur. Acad. Dermatol. Venereol. 3(suppl):S23-31.
72. TOPERT, M. 1988. Perspectives in corticosteroid research. Drugs. 36(suppl 5):38-42.

73. TRICKER, R.; CASABURI, R.; STORER, T.W.; CLEVENGER, B.; BERMAN, N.; SHIRAZI, A.; BHASIN, S. 1996. The effects of supraphysiological doses of testosterone on angry behavior in healthy eugonadal men-a clinical research center study. J. Clin. Endocr. Metab. 81:3754-3758.

74. TUREK, P.J.; WILLIAMS, R.H., GILBAUCH, J.H. 3rd; LIPSHULTZ, L.I. 1995. The reversibility. of anabolic steroid-induced azospermia. J. Urol. 153:16281630.

75. WILSON, J.D. 1988. Androgen abusy by athletes. Endocr. Rev. 9:189-199.

76. WORLD ANTI-DOPING AGENCY WADA. 2009. World Antidoping Code, Quebec. p.5-10.

77. YESALIS, C.E.; COWART, V.S. 1999. Esteroides, un juego peligroso. Ed. Hispano Europea. Barcelona. $199 p$.

78. YESALIS, C.E.; BAHRKE, M.S. 1995. Anabolicandrogenic steroids. Current issues. Sports Med. 19(5):326-340.

79. ZITZMANN, M.; BRUNE, M.; NIESCHLAG, E. 2002. Vascular reactivity in hypogonadal men is reduced by androgen substitution. J. Clin. Endocr. Metab. 87:5030-5037.

Recibido: Enero 18 de 2012

Aceptado: Marzo 15 de 2012 